\title{
Desarrollo inicial de ateroesclerosis en aorta de conejos sometidos a dietas con exceso de colesterol
}

\author{
Lértora, W.J.'; Villordo, G.I. ; Mussart, N.B. ${ }^{2}$; Catuogno, M.S.'; Sánchez Negrette, M. ${ }^{1}$ \\ ${ }^{1}$ Cátedra de Patología General y Sistemática, ${ }^{2}$ Cátedra de Fisiología, Facultad de Ciencias Veterinarias, \\ Universidad Nacional del Nordeste, Sargento Cabral 2139, Corrientes (3400), Argentina. Tel-Fax: +54 (379) \\ 4425753. \\ E-mail: patgral@vet.unne.edu.ar
}

\begin{abstract}
Resumen
Lértora, W.J.; Villordo, G.I.; Mussart, N.B.; Catuogno, M.S.; Sánchez Negrette, M.: Desarrollo inicial de ateroesclerosis en aorta de conejos sometidos a dietas con exceso de colesterol. Rev. vet. 25: 2, 87-94, 2014. Para obtener un modelo experimental de ateroesclerosis aórtica, se suministraron tres dosis de colesterol dietético $(0,62 ; 0,93$ y $1,24 \mathrm{~g} /$ día) durante 60 días a conejos neozelandeses machos adultos. Se evaluaron los niveles séricos de colesterol, colesterol-HDL y LDL, aspartato aminotransferasa (AST) y alanin aminotransferasa (ALT), así como el desarrollo de ateroesclerosis en aorta. El incremento en la dosis de 0,62 a $0,93 \mathrm{~g} /$ día de colesterol ocasionó un aumento significativo $(\mathrm{p} \leq 0,05)$ en la colesterolemia (de $6,79 \pm 2,34$ a 9,51 $\pm 0,10 \mathrm{~g} / \mathrm{l})$, principalmente por incremento del colesterol-LDL. La dosis de $1,24 \mathrm{~g} /$ día no indujo aumento adicional de la colesterolemia comparado con la dosis de $0,93 \mathrm{~g} /$ día. La colesterolemia fue muy variable entre los conejos que recibieron $0,62 \mathrm{~g} /$ día; las dosis mayores de colesterol redujeron esta variabilidad. Las actividades ALT y AST permanecieron normales hasta el día 30 de la experiencia, incrementándose significativamente en el día $60(\mathrm{p} \leq 0,05)$ en los grupos que recibieron 0,93 y $1,24 \mathrm{~g} / \mathrm{d}$ á, asociadas a degeneración grasa hepática. Las tres dosis de colesterol indujeron áreas sudanofílicas de extensión variable en aorta. Las lesiones en aorta se caracterizaron por un incremento focal del espesor intimal causado por la infiltración de macrófagos espumosos y músculo liso, entremezclados en proporción variable con depósitos de matriz extracelular. En conclusión, se confirma la sensibilidad del conejo neozelandés a desarrollar hipercolesterolemia y lesiones ateroescleróticas en un corto período de tiempo con una dieta rica en colesterol. La dosis de $0,93 \mathrm{~g} /$ día durante 60 días fue la que indujo una respuesta hipercolesterolémica con menor variabilidad entre los conejos y lesiones ateroescleróticas bien desarrolladas. El modelo experimental de ateroesclerosis temprana permitirá estudiar el efecto ateroprotector de diferentes medicamentos y alimentos funcionales.
\end{abstract}

Palabras clave: conejo, modelo experimental, aorta, ateroesclerosis, colesterol.

\begin{abstract}
Lértora, W.J.; Villordo, G.I.; Mussart N.B.; Catuogno M.S.; Sánchez Negrette M.: Initial stage of atherosclerosis in aorta of rabbits by administering cholesterol. Rev. vet. 25: 2, 87-94, 2014. To obtain an experimental model of aortic atherosclerosis, male adult New Zealand rabbits were fed with three doses of dietary cholesterol $(0.62,0.93$ and $1.24 \mathrm{~g} /$ day $)$ for 60 days. Serum cholesterol, HDL and LDL-cholesterol, aspartate aminotransferase (AST), alanin aminotransferase (ALT) and the development of atherosclerosis in aorta, were evaluated. Increased dietary cholesterol from 0.62 to $0.93 \mathrm{~g} /$ day resulted in a significant increase $(\mathrm{p} \leq 0.05)$ in serum cholesterol (from $6.79 \pm 2.34$ to $9.51 \pm 0.10 \mathrm{~g} / \mathrm{l})$, due mainly to the increase in LDL-cholesterol. The dose of $1.24 \mathrm{~g} /$ day did not induce further increase in cholesterolemia compared to the dose of $0.93 \mathrm{~g} /$ day. Serum cholesterol was highly variable among rabbits that received $0.62 \mathrm{~g} /$ day, this variability was reduced with the highest dose of cholesterol. ALT and AST activities remained normal until day 30 of experience, increasing significantly at day $60(p \leq 0.05)$ in the groups receiving cholesterol at the doses of 0.93 and $1.24 \mathrm{~g} /$ day, associated with liver fatty degeneration. All doses of cholesterol induced aortic sudanophilic lesion areas of variable surface. Aortic lesions were characterized by a focal increase in intima thickening caused by infiltration of foamy macrophages and smooth muscle mixed in vari-
\end{abstract}


able proportions with extracellular matrix deposition. In conclusion, the sensitivity of the New Zealand rabbit to develop hypercholesterolemia and atherosclerotic lesions in a short period of time when consuming a high cholesterol diet, is confirmed. The dose of $0.93 \mathrm{~g} / \mathrm{day}$ for 60 days caused a lower variability in the hypercholesterolemic response among rabbits with significant development of atherosclerotic lesions. This model of early atherosclerosis will allow the study of the atheroprotective effects of different medicines and functional foods.

Key words: rabbit, experimental model, aorta, atherosclerosis, cholesterol.

\section{INTRODUCCIÓN}

Debido a su amplia distribución, la ateroesclerosis es la enfermedad de mayor morbilidad y mortalidad en el mundo occidental, siendo responsable de aproximadamente la mitad de todos los fallecimientos ${ }^{23}$. En Argentina, las enfermedades cardiovasculares son la primera causa de muerte entre la población general y una importante causa de discapacidad ${ }^{21}$.

Para identificar factores nutricionales y nuevas drogas que inhiban el desarrollo de la ateroesclerosis o sus complicaciones, resulta necesario emplear modelos biológicos experimentales. Ratones ${ }^{14}$, conejos ${ }^{27}$, porcinos $^{6}$, hámsteres ${ }^{7}$, cobayos ${ }^{18}$, palomas y primates no humanos ${ }^{20}$ han sido utilizados como modelos de ateroesclerosis. La mayoría de estos animales desarrollan hipercolesterolemia y ateroesclerosis mediante manipulación dietética o genética.

La elección del modelo animal de ateroesclerosis dependerá del propósito de la investigación ${ }^{13}$. Si se desea estudiar el estadio inicial de la ateroesclerosis, caracterizado por infiltración de macrófagos espumosos en la capa íntima arterial (estría grasa) y otros aspectos de la inflamación arterial, se puede emplear al "conejo alimentado con colesterol". El conejo neozelandés es muy sensible al colesterol dietético desarrollando una severa hipercolesterolemia y lesión ateroesclerótica temprana en un corto período de tiempo. La concentración de colesterol y la duración de su administración dependen de cada estudio. Una dieta con $0,25 \%$ de colesterol durante 4 semanas es suficiente para estudiar los estadios iniciales de la formación de estrías grasas ${ }^{28}$.

Empleando dietas con $1 \%$ de colesterol durante 8 a 12 semanas, en otras investigaciones se logró la formación de estrías grasas bien desarrolladas, con abundantes células espumosas derivadas de macrófagos ${ }^{2,4,19}$. Para estudiar lesiones ateroescleróticas más avanzadas (placa fibrograsosa) se emplearon dosis más bajas y lapsos más prolongados $(0,25 \%$ de colesterol durante 8 meses) ${ }^{16}$.

Con el objetivo de estandarizar un modelo experimental de ateroesclerosis temprana en conejos neozelandeses, se evaluaron los efectos de tres dosis de colesterol sobre la colesterolemia, los niveles séricos de aspartato aminotransferasa y alanin aminotransferasa, el desarrollo de ateroesclerosis en aorta y los cambios histopatológicos de diversos órganos.

\section{MATERIAL Y MÉTODOS}

Animales y plan experimental. Todos los procedimientos de este estudio fueron aprobados por el Comité de Ética y Bioseguridad de la Facultad de Ciencias Veterinarias de la UNNE, protocolo $\mathrm{N}^{\circ}$ 017. Se emplearon 24 conejos neozelandeses machos adultos, con un peso de 2,154 $\pm 0,289 \mathrm{~kg}$ obtenidos del Centro de Experimentaciones Biológicas y Bioterio de la Universidad Nacional del Litoral. Fueron alojados en jaulas metálicas individuales, con una temperatura ambiente de $22^{\circ} \mathrm{C}$, un ciclo de luz/oscuridad de 12 horas, con agua de bebida y alimento balanceado ad libitum. Luego de un período de adaptación de 15 días los animales fueron divididos en cuatro grupos: control $(n=6$, que recibió $2 \mathrm{ml}$ de placebo/día), grupo 1 ( $\mathrm{n}=6$, recibió $2 \mathrm{ml} /$ día de jarabe con colesterol [94\%], equivalente a $0,62 \mathrm{~g} / \mathrm{día}$ de colesterol); grupo 2 ( $\mathrm{n}=6$, recibió $3 \mathrm{ml} /$ día del jarabe con colesterol, equivalente a $0,93 \mathrm{~g} /$ día) y grupo $3(\mathrm{n}=6$, recibió $4 \mathrm{ml} /$ día del jarabe (1,24 g/día de colesterol). El jarabe con colesterol y el placebo se administraron durante 60 días por vía oral, utilizando una jeringa de 1 $\mathrm{ml}$, mediante la cual se introdujo la dosis directamente en la cavidad bucal.

Preparación del colesterol y placebo. El colesterol (Cholesterol 94\%, Sigma Chemical Co., St. Louis, USA) fue administrado en forma de jarabe simple según la siguiente fórmula: colesterol al $94 \%=200 \mathrm{~g}$, alcohol etílico $=160 \mathrm{ml}$, jarabe simple $=320 \mathrm{ml}$ y conservantes $=2 \mathrm{ml}$ (metilparabeno $6 \mathrm{~g}$, propilparabeno 14 $\mathrm{g}$ y propilenglicol $180 \mathrm{~g}$ ). Cada mililitro de esta preparación contenía $0,31 \mathrm{~g}$ de colesterol al 94\%. El placebo consistió en una combinación de alcohol etílico (160 $\mathrm{ml})$, jarabe simple $(320 \mathrm{ml})$ y conservantes $(2 \mathrm{ml})$.

Evaluaciones durante la experiencia. Todos los animales fueron examinados diariamente a fin de detectar modificaciones físicas o de comportamiento. El peso corporal fue registrado al inicio del experimento y luego semanalmente en todos los animales de los diferentes grupos. La extracción de sangre se realizó a los 0 , 30 y 60 días, bajo ayuno de 8 horas, a partir de la arteria auricular, empleando aguja $25 \mathrm{G}$ x $5 / 8^{\prime}$ y jeringas de $5 \mathrm{ml}$, bajo anestesia general (combinación de ketamina $35 \mathrm{mg} / \mathrm{kg}$ y xilacina $5 \mathrm{mg} / \mathrm{kg}$, vía subcutánea). Mediante espectrofotometría, con reactivos Wiener Lab se 
efectuaron determinaciones séricas de colesterol total (colesterol-oxidasa-peroxidasa, $505 \mathrm{~nm}$ ) y colesterol ligado a lipoproteínas de alta y baja densidad (C-HDL y C-LDL), por precipitación selectiva de la lipoproteína y valoración enzimática de colesterol. Como marcadores de daño hepático se determinaron las actividades de aspartato aminotransferasa AST (técnica aspartato cetoglutarato) y alanin aminotransferasa ALT (técnica alanina cetoglutarato), con lectura a $505 \mathrm{~nm}$ en espectrofotómetro Zeltec ZL 5000.

Necropsia y toma de muestras. A los 60 días de iniciada la experiencia, se procedió a la eutanasia y necropsia de los animales. Se obtuvieron muestras de aorta completa, corazón, pulmón, hígado, riñón, bazo, cerebro y ojo, las que fueron fijadas en formol bufferado al 10\% durante 24 horas para su análisis macroscópico e histológico.

Cuantificación del área sudanofilica de la aorta. Luego de la fijación, las aortas completas fueron procesadas para la coloración macroscópica de los depósitos lipídicos en el estrato subintimal. Cada aorta fue disecada desde sus válvulas hasta la bifurcación ilíaca. Se removió cualquier exceso de tejido y grasa adherido a la adventicia y posteriormente se realizó una coloración para lípidos con una solución de Sudan IV ${ }^{12}$. Luego, cada aorta se extendió sobre una lámina de tergopol con la superficie endotelial hacia arriba y se fotografío la superficie endotelial con una cámara digital (Canon EOS Digital Rebel xsi) sujeta a un estativo. Las imágenes digitales fueron editadas para eliminar el fondo y segmentadas en áreas sudanofílicas y no sudanofílicas, ajustando manualmente el control de umbralización de color al umbral que mejor representara la lesión sudanofílica. Para esta tarea, se empleó el software ImageJ versión 1.47 n, U.S. National Institutes of Health, Bethesda, Maryland, USA. El área sudanofílica de cada aorta fue expresada en porcentaje de la superficie intimal cubierta por la lesión sudanofílica.

Histomorfometría de la aorta. Luego de ser fotografiada, una sección de $5 \mathrm{~mm}$ de la aorta ascendente, en craneal del tronco braquiocefálico, fue procesada según la técnica histológica clásica para bloques parafinados. Con un micrótomo, cada $5 \mu \mathrm{m}$ se realizaron cortes seriados que fueron coloreados con hematoxilina y eosina (H-E) e inmunoteñidos con el anticuerpo monoclonal anti-actina muscular para identificar músculo liso vascular (HHF-35, Dako) y con el anticuerpo monoclonal anti-macrófagos de conejos (RAM11, Dako). En las secciones teñidas con H-E se midió en micrómetros, con objetivo de 10x, el espesor de la túnica íntima (desde la superficie luminal hasta la membrana elástica interna) en el sitio de máximo espesor intimal. En las secciones inmunoteñidas, las lesiones de la aorta se clasificaron en estría grasa (intima con infiltrado de macrófagos espumosos), placa en transición (intima con infiltrado mixto de macrófagos espu- mosos, músculo liso y depósito de matriz extracelular) y ateroma (intima con capa fibrosa de músculo liso y matriz extracelular cubriendo un centro de macrófagos espumosos con focos de necrosis y cristales de colesterol extracelular).

Histopatología de órganos. Corazón, pulmón, hígado, riñón, bazo, cerebro y ojo fueron procesados según la técnica histológica clásica para bloques parafinados, cortados a $5 \mu \mathrm{m}$ y coloreados con H-E para su análisis histopatológico.

Análisis estadístico. Los resultados cuantitativos se procesaron bajo un diseño experimental aleatorizado. Las estadísticas descriptivas incluyeron media aritmética y desvío estándar ( $\overline{\mathrm{x}} \pm \mathrm{DE})$. Los datos inferenciales fueron obtenidos por análisis de la varianza (ANOVA) a una vía y las diferencias entre grupos fueron comparadas por el test de Tukey. El nivel del riesgo alfa se fijó en 5\%, por debajo del cual se rechazó la hipótesis nula de igualdad.

\section{RESULTADOS Y DISCUSIÓN}

Administración del colesterol. Todos los conejos aceptaron sin inconvenientes las diferentes dosis de colesterol vehiculizado en el jarabe. La mayoría de los modelos biológicos de ateroesclerosis que emplean dietas hipercolesterolémicas adicionan el colesterol, en diferentes proporciones, al alimento balanceado $2,4,11,19,28$. En este trabajo se optó por administrar el colesterol vía bucal para asegurar que cada conejo consuma la dosis diaria correspondiente. Probablemente, el sabor dulce del jarabe facilitó que los animales aceptaran sin inconvenientes la dosis diaria de colesterol.

Evaluación general, del peso corporal y del peso del hígado. Los conejos que recibieron colesterol en sus diferentes dosis y placebo ganaron peso a lo largo de la experiencia. La ganancia de peso corporal y el peso del hígado no difirió significativamente $(\mathrm{p}=$ 0,6417 y $p=0,6747$, respectivamente) entre los conejos que recibieron colesterol y placebo (Tabla 1).

Tabla 1. Ganancia de peso corporal y peso del hígado según grupo.

\begin{tabular}{lcc}
\hline grupo & ganancia de peso $(\mathrm{kg})$ & peso del hígado $(\mathrm{g})$ \\
\hline control & $1,08 \pm 0,36^{\mathrm{a}}$ & $138,33 \pm 29,27^{\mathrm{a}}$ \\
grupo 1 & $1,01 \pm 0,21^{\mathrm{a}}$ & $145,00 \pm 24,29^{\mathrm{a}}$ \\
grupo 2 & $0,87 \pm 0,32^{\mathrm{a}}$ & $136,67 \pm 31,41^{\mathrm{a}}$ \\
grupo 3 & $0,96 \pm 0,25^{\mathrm{a}}$ & $153,33 \pm 15,06^{\mathrm{a}}$ \\
\hline
\end{tabular}

Datos en $\overline{\mathrm{X}} \pm$ DE. ${ }^{\mathrm{a}}$ : medias con una letra común no son significativamente diferentes $(\mathrm{p}>0,05)$.

En nuestra experiencia, la administración de 0,62 , 0,93 o $1,24 \mathrm{~g}$ /día de colesterol durante 60 días no afectó la ganancia de peso corporal y no indujo cambios físicos o de comportamiento en los conejos. Estos re- 
sultados concuerdan con los obtenidos en experiencias cortas (2-3 meses) que emplearon dietas con 0,5 a $1 \%$ de colesterol para la inducción de ateroesclerosis ${ }^{4,26}$. Por el contrario, la administración prolongada (31-32 semanas) de una dieta hipercolesterolémica $(0,25 \%$ de colesterol) provocó reducción en el consumo de alimento y una significativa pérdida de peso corporal al final de la experiencia, debido a la hepatotoxicidad del colesterol ${ }^{16}$. En la presente investigación, el colesterol tampoco afectó el peso del hígado, a diferencia de trabajos que reportan un incremento en los pesos del hígado, corazón y riñón en conejos que consumieron alimento con 0,7 a $1 \%$ de colesterol durante 60 días $^{4}$.

Colesterolemia. La Tabla 2 registra los niveles de colesterol total, C-HDL y C-LDL de los diferentes grupos a lo largo de la experiencia. Al inicio (día 0), los niveles de los tres parámetros no difirieron entre grupos. En el día 30 del ensayo, los conejos que recibieron colesterol elevaron significativamente los niveles de colesterol total y C-LDL. Los niveles basales de C-LDL incrementaron 12 veces con la dosis de $0,62 \mathrm{~g} /$ día (grupo 1), mientras que el incremento fue de 22 veces con la dosis de $0,93 \mathrm{~g} /$ día (grupo 2). La dosis mayor de colesterol en el grupo 3 no provocó un incrementó proporcional, comparado con el grupo 2, en los niveles de C-LDL y colesterol total. Hasta el final del estudio (día 60), los niveles séricos de colesterol total y C-LDL se mantuvieron sin diferencias, con respecto al día 30, en los tres grupos tratados. El incremento en los niveles séricos de C-HDL fue menor al incremento registrado para C-LDL en los tres grupos tratados con colesterol a lo largo de la experiencia. En los días 30 y 60 de la experiencia, los conejos que recibieron 0,93 y $1,24 \mathrm{~g} /$ día de colesterol fueron los que incrementaron significativamente sus niveles de C-HDL, con respecto

Tabla 2. Evolución de parámetros relacionados al colesterol según grupo.

\begin{tabular}{lccc}
\hline tasa sérica $(\mathrm{g} / \mathrm{l})$ & día 0 & día 30 & día 60 \\
\hline $\begin{array}{l}\text { colesterol total } \\
\text { control }\end{array}$ & $0,92 \pm 0,29^{\mathrm{a}}$ & $0,94 \pm 0,32^{\mathrm{a}}$ & $0,60 \pm 0,09^{\mathrm{a}}$ \\
grupo 1 & $0,95 \pm 0,39^{\mathrm{a}}$ & $5,97 \pm 2,42^{\mathrm{b}}$ & $6,79 \pm 2,34^{\mathrm{b}}$ \\
grupo 2 & $0,88 \pm 0,24^{\mathrm{a}}$ & $8,99 \pm 0,25^{\mathrm{c}}$ & $9,51 \pm 0,10^{\mathrm{c}}$ \\
grupo 3 & $1,01 \pm 0,12^{\mathrm{a}}$ & $9,17 \pm 0,21^{\mathrm{c}}$ & $9,48 \pm 0,08^{\mathrm{c}}$ \\
\hline colesterol-HDL & & & \\
control & $0,54 \pm 0,08^{\mathrm{a}}$ & $0,41 \pm 0,11^{\mathrm{a}}$ & $0,32 \pm 0,15^{\mathrm{a}}$ \\
grupo 1 & $0,35 \pm 0,06^{\mathrm{a}}$ & $0,46 \pm 0,07^{\mathrm{a}}$ & $1,23 \pm 0,77^{\mathrm{a}}$ \\
grupo 2 & $0,40 \pm 0,10^{\mathrm{a}}$ & $1,78 \pm 1,22^{\mathrm{b}} \mathrm{c}$ & $2,57 \pm 0,45^{\mathrm{c}}$ \\
grupo 3 & $0,33 \pm 0,06^{\mathrm{a}}$ & $2,72 \pm 1,04^{\mathrm{c}}$ & $2,39 \pm 0,64^{\mathrm{c}}$ \\
\hline colesterol-LDL & & \\
control & $0,24 \pm 0,11^{\mathrm{a}}$ & $0,32 \pm 0,15^{\mathrm{a}}$ & $0,18 \pm 0,06^{\mathrm{a}}$ \\
grupo 1 & $0,29 \pm 0,18^{\mathrm{a}}$ & $3,52 \pm 2,10^{\mathrm{b}}$ & $4,10 \pm 2,10^{\mathrm{b}}$ \\
grupo 2 & $0,27 \pm 0,12^{\mathrm{a}}$ & $6,15 \pm 0,25^{\mathrm{c}}$ & $6,65 \pm 0,15^{\mathrm{c}}$ \\
grupo 3 & $0,43 \pm 0,08^{\mathrm{a}}$ & $6,33 \pm 0,21^{\mathrm{c}}$ & $6,65 \pm 0,13^{\mathrm{c}}$ \\
\hline
\end{tabular}

Datos en $\overline{\mathrm{X}} \pm$ DE. ${ }^{\mathrm{a}, \mathrm{b}, \mathrm{c}}$ : en cada columna, letras distintas indican diferencias significativas $(\mathrm{p} \leq 0,05)$. al grupo control. Los conejos que recibieron placebo mantuvieron los niveles de colesterol total, C-HDL y C-LDL en valores normales y estables durante toda la experiencia.

Consumiendo su dieta normal, el conejo neozelandés registra bajos niveles séricos de colesterol total, C-LDL, C-HDL y C-VLDL (colesterol de lipoproteínas de muy baja densidad). Luego de una dieta con colesterol, sus valores de colesterol total, C-LDL y C-VLDL se elevan significativamente, manteniendo bajos sus niveles de C-HDL 11, 16, 24, 28 . Esta hipercolesterolemia, principalmente por incremento del colesterol transportado por lipoproteínas aterogénicas, se debe a un descenso en la tasa de eliminación metabólica de las lipoproteínas LDL y $\beta$-VLDL. La hipercolesterolemia induce una saturación y supresión de receptores de $\beta$-VLDL en el hígado de los conejos. Como resultado, la eliminación hepática de $\beta$-VLDL está disminuida, no aumenta en concordancia con el aumento en la síntesis de $\beta$-VLDL, incrementando los niveles y tiempo de permanencia en la circulación sanguínea del colesterol asociado a lipoproteínas aterogénicas ${ }^{17}$. El incremento en los niveles y tiempo de permanencia de las lipoproteínas aterogénicas en sangre induce el desarrollo de ateroesclerosis en este modelo ${ }^{16}$.

La colesterolemia se incrementa proporcionalmente a la dosis de colesterol dietético consumido ${ }^{16}$. En nuestra experiencia, la hipercolesterolemia fue menor en el grupo que recibió $0,62 \mathrm{~g}$ /día y fue mayor en los conejos que recibieron $0,93 \mathrm{~g} /$ día, mientras que la dosis de $1,24 \mathrm{~g}$ /día no incrementó proporcionalmente la colesterolemia. La dosis de $0,93 \mathrm{~g} /$ día pudo haber ocasionado una saturación de las lipoproteínas aterogénicas y la dosis mayor, al superar el umbral de saturación, no incrementó proporcionalmente la colesterolemia.

Se observaron variaciones individuales en los niveles de colesterol en el grupo que recibió $0,62 \mathrm{~g}$ /día: en el día 30 se registraron valores mínimos de 3,22 g/l y máximos de $8,96 \mathrm{~g} / 1$ para colesterol total, así como mínimos de $1,28 \mathrm{~g} / 1$ y máximos de $6,14 \mathrm{~g} / 1$ para C-LDL (datos no mostrados en Tabla 2). Además, en el día 60 , los niveles de colesterol total variaron de 2,75 a 9,31 g/1 y C-LDL registró mínimas de 0,69 y máximas de $6,27 \mathrm{~g} / 1$ (datos no mostrados en Tabla 2). Por el contrario, los niveles de colesterolemia fueron menos variables entre los conejos que recibieron dosis de $0,93 \mathrm{y}$ 1,24 g/día. Los 12 conejos de ambos grupos registraron, en los días 30 y 60 de la experiencia, niveles mínimos de 8,65 y máximos de $9,68 \mathrm{~g} / 1$ de colesterol total y valores mínimos de 5,83 g/l y máximos de $6,85 \mathrm{~g} / 1$ de C-LDL (datos no mostrados en Tabla 2).

Esta variabilidad en los niveles de colesterolemia en respuesta a una misma dosis de colesterol dietético es inherente del conejo. Puede ser controlada ajustando la dosis de colesterol o preseleccionando conejos con similar sensibilidad al colesterol dietético. Además, incrementar el número de conejos empleados en la experiencia minimiza la variabilidad individual propia de este modelo ${ }^{16}$. En nuestro ensayo, las dosis de 0,93 
Tabla 3. Evolución de las actividades séricas de ALT y AST, según grupo.

\begin{tabular}{|c|c|c|c|}
\hline tasa sérica $(\mathrm{U} / \mathrm{l})$ & día 0 & día 30 & día 60 \\
\hline \multicolumn{4}{|l|}{$\overline{\operatorname{ALT}(\mathrm{U} / \mathrm{l})}$} \\
\hline control & $11,00 \pm 9,53^{\mathrm{a} b}$ & $8,83 \pm 4,49^{a b}$ & $5,33 \pm 3,50^{\mathrm{a}}$ \\
\hline grupo 1 & $9,83 \pm 4,02^{\mathrm{a} \mathrm{b}}$ & $6,83 \pm 2,14^{\mathrm{a} b}$ & $17,33 \pm 7,69^{a b c}$ \\
\hline grupo 2 & $10,33 \pm 4,97^{\mathrm{a} b}$ & $8,83 \pm 4,54^{\mathrm{a} b}$ & $29,17 \pm 11,07^{\mathrm{cd}}$ \\
\hline grupo 3 & $18,33 \pm 6,15^{\mathrm{bc}}$ & $6,50 \pm 3,56^{\mathrm{a} b}$ & $37,17 \pm 9,72^{\mathrm{d}}$ \\
\hline \multicolumn{4}{|l|}{$\overline{\operatorname{AST}(U / 1)}$} \\
\hline control & $9,83 \pm 5,27^{\mathrm{a} b}$ & $10,0 \pm 5,55^{\mathrm{a} b}$ & $6,17 \pm 4,12^{\mathrm{a} b}$ \\
\hline grupo 1 & $5,50 \pm 1,64^{a}$ & $8,83 \pm 2,56^{\mathrm{ab}}$ & $6,50 \pm 1,22^{\mathrm{a} b}$ \\
\hline grupo 2 & $4,17 \pm 2,32^{\mathrm{a}}$ & $4,33 \pm 1,97^{\mathrm{a}}$ & $14,33 \pm 8,24^{b c}$ \\
\hline grupo 3 & $8,67 \pm 3,33^{a b}$ & $6,50 \pm 5,75^{\mathrm{ab}}$ & $20,33 \pm 4,08^{c}$ \\
\hline
\end{tabular}

Datos en $\overline{\mathrm{X}} \pm$ DE. ${ }^{\mathrm{a}, \mathrm{b}, \mathrm{c}, \mathrm{d}}$ : en cada columna, letras distintas indican diferencias significativas $(\mathrm{p} \leq 0,05)$.

y $1,24 \mathrm{~g} /$ día parece haber controlado esta variabilidad biológica, con respecto a la respuesta individual al colesterol dietético, característica del conejo.

Enzimograma. La Tabla 3 registra los valores séricos de ALT y AST en los cuatro grupos a lo largo de la experiencia. En los conejos que recibieron colesterol (grupos 1, 2 y 3), los niveles de ALT permanecieron normales hasta el día 30, incrementándose significativamente en el día 60. El incremento fue mayor en los conejos que recibieron 1,24 g/día (grupo 3). Los niveles de AST se mantuvieron normales hasta el día 30, incrementándose significativamente el día 60 en los conejos que recibieron 0,93 y 1,24 g/día (grupos 2 y 3). Los niveles de AST permanecieron esencialmente normales en los conejos que recibieron 0,62 g/día (grupo 1). Los niveles de las enzimas ALT y AST en los conejos del grupo control permanecieron normales a lo largo de la experiencia.

Todos los conejos que recibieron colesterol desarrollaron degeneración grasa hepática, lesión que se podría relacionar con el incremento en los niveles de ALT y AST. En ratones, elevaciones de ALT y AST fueron vinculadas con la degeneración grasa hepática provocada por dietas ricas en lípidos, con simultánea resistencia a la insulina, estrés oxidativo y disfunción mitocondrial ${ }^{30}$. Otros trabajos demostraron que dosis elevadas de colesterol o su administración prolongada provocan hepatotoxicidad. Conejos alimentados con dietas conteniendo más de $0,15 \%$ de colesterol presentaron incrementos de ALT y AST después de 12 semanas de exposición ${ }^{16}$. Dietas con $1 \%$ de colesterol durante 3 meses causaron esteatosis y fibrosis hepática en conejos, con incremento de las enzimas hepáticas al mes de exposición ${ }^{19}$. Sin embargo, pese a la hepatotoxicidad del colesterol, muchos estudios emplean este modelo para probar el efecto de diversas drogas y alimentos en el desarrollo de la ateroesclerosis $2,4,11,19,27$.

En seres humanos, el incremento de las actividades ALT y AST se asocia con desordenes hepáticos, incluyendo esteatosis, inducidos por componentes del sín- drome metabólico (resistencia a la insulina, obesidad central, dislipidemia e hipertensión arterial). El síndrome metabólico incrementa notablemente el riesgo de sufrir ateroesclerosis y diabetes tipo 2. Varios estudios han demostrado que los niveles de transaminasas hepáticas, principalmente ALT, pueden ser utilizados como un marcador para predecir el riesgo de ateroesclerosis coronaria y diabetes en seres humanos ${ }^{1,22,29}$.

Area sudanofilica en aorta. Todos los conejos que recibieron colesterol desarrollaron lesiones sudanofílicas en aorta. Las extensiones de las áreas sudanofílicas en los grupos 1, 2 y 3 (Tabla 4) no presentaron diferencias significativas $(p=0,6891)$. Los conejos del grupo control no desarrollaron lesiones en aorta.

Se afirma la existencia de correlación positiva entre los niveles de colesterol plasmático y el área sudanofíli$\mathrm{ca}^{16}$. En la presente experiencia, la alta variabilidad del área sudanofílica, el bajo número de conejos empleados y/o la dosis de colesterol utilizada no permitieron apreciar esta asociación. Sin embargo, se constató una tendencia al desarrollo de lesiones sudanofílicas menos extensa en el grupo que recibió menos colesterol y lesiones sudanofílicas de mayor dimensión en los conejos que recibieron 0,93 y 1,24 g/día. Esta tendencia pudo deberse a la magnitud de la colesterolemia, que fue menor en el grupo que recibió $0,62 \mathrm{~g} /$ día y mayor en los grupos que recibieron 0,93 y $1,24 \mathrm{~g} /$ día. La dosis de $0,93 \mathrm{~g}$ /día pudo haber ocasionado una saturación de las lipoproteínas aterogénicas y la dosis mayor, al superar el umbral de saturación, no habría incrementado proporcionalmente la colesterolemia y el área sudanofílica.

Se observó variabilidad en la extensión sudanofílica entre los conejos dentro de los tres grupos. Con la dosis de 0,62 g/día se observaron extensiones mínimas de $0,88 \%$ y máximas de $75,53 \%$ en la lesión sudanofílica. Con la dosis de $0,93 \mathrm{~g} /$ día la extensión sudanofílica registró mínimas de 14,38\% y máximas de 86,96\%; mientras que a mayor dosis (1,24 g/día) la mínima fue de $9,36 \%$ y la máxima $65,21 \%$. La variabilidad en la extensión de las lesiones ateroescleróticas ante una misma dosis de colesterol dietético y a un mismo nivel de colesterolemia es coincidente con los resultados reportados por otros investigadores ${ }^{24}$. Ante hipercolesterolemias inducidas por dietas, la alta y baja magnitud de las respuestas del conejo fueron atribuidas a factores genéticos ${ }^{24}$. Seres humanos hipercolesterolémicos

Tabla 4. Área sudanofílica y espesor intimal en aorta, según grupo.

\begin{tabular}{lcc}
\hline grupo & área sudanofílica $(\%)$ & espesor intimal $(\mu \mathrm{m})$ \\
\hline 1 & $24,07 \pm 28,43^{\mathrm{a}}$ & $116,85 \pm 55,88^{\mathrm{a}}$ \\
2 & $36,15 \pm 26,41^{\mathrm{a}}$ & $201,56 \pm 103,41^{\mathrm{ab}}$ \\
3 & $35,79 \pm 26,87^{\mathrm{a}}$ & $268,68 \pm 88,81^{\mathrm{b}}$ \\
\hline
\end{tabular}

Datos en $\overline{\mathrm{x}} \pm$ DE. ${ }^{\mathrm{a}, \mathrm{b}}$ : en cada columna, letras distintas indican diferencias significativas $(\mathrm{p} \leq 0,05)$. 
también poseen factores genéticos que influencian la expresión de las lesiones ateroescleróticas en la aorta ${ }^{10}$.

Las lesiones sudanofílicas se localizaron principalmente en el origen de la aorta y en los orificios de las ramificaciones arteriales; dicha localización fue similar en todos los conejos que recibieron colesterol. En la aorta ascendente se localizaron en la pared dorsal del arco aórtico, rodeando los orificios de origen del tronco braquiocefálico y de la arteria subclavia izquierda. En las porciones torácica y abdominal de la aorta, las lesiones sudanofílicas se ubicaron en la mitad caudal de los orificios de las arterias intercostales, celíacas, mesentéricas, renales y lumbares. En estos lugares la lesión sudanofílica tuvo forma de cabeza de flecha con la punta dirigida hacia caudal (Figura 1).

Todos los conejos que recibieron colesterol presentaron lesiones sudanofílicas con la típica localización reportada en los modelos de ateroesclerosis experimental que emplean dietas hipercolesterolémicas ${ }^{3,6,16}$. Cerdos alimentados durante 9 meses con dieta aterogénica $(15 \%$ de grasa y $1,5 \%$ de colesterol) revelaron mayor sudanofília en el origen de la aorta (alrededor de los orificios de las coronarias, orificios del tronco braquiocefálico y de la arteria subclavia izquierda, curvatura externa del arco aórtico y en distal a la cicatriz del conducto arterioso), en los bordes de los orificios

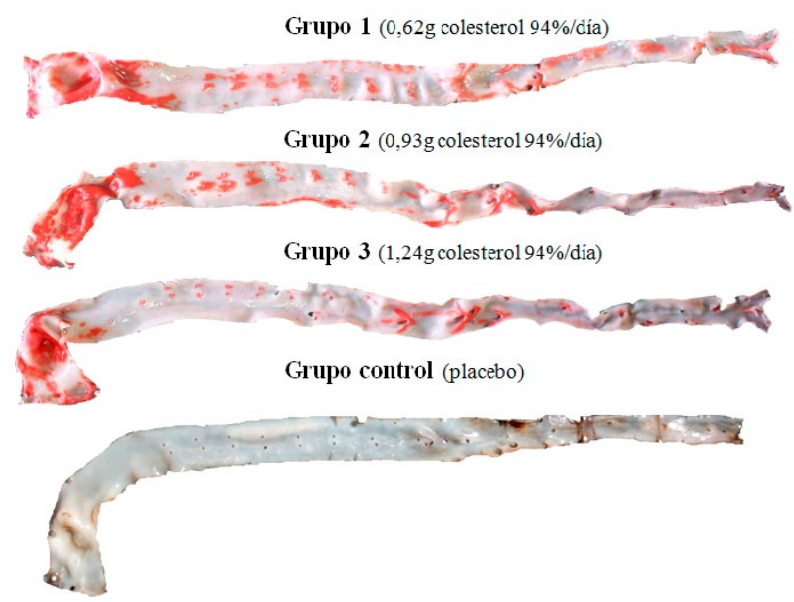

Figura 1. Localización de lesiones sudanofílicas en aortas de conejos sometidos a dosis crecientes de colesterol dietético.
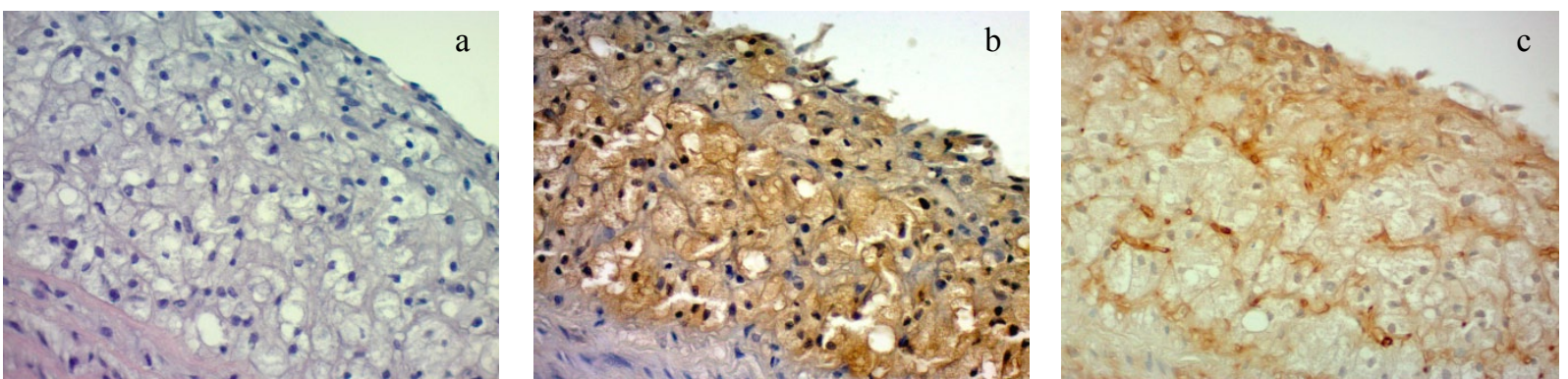

Figura 2. Fotomicrografías de aorta con lesión ateroesclerótica de un conejo tratado con $1,24 \mathrm{~g}$ colesterol/día. Cortes seriados: teñido con H-E (a), inmunoreactivo al anticuerpo anti-macrófago (b) e inmunoreactivo al anticuerpo anti-actina de músculo liso (c). Se aprecia infiltrado intimal de abundantes macrófagos espumosos y, en menor proporción, músculo liso (40x). 
mayores dosis de colesterol desarrollaron mayor hipercolesterolemia y lesiones de mayor espesor en aorta ${ }^{16}$.

Morfología de lesiones ateroescleróticas. Todos los conejos que recibieron colesterol (grupos 1, 2 y 3) desarrollaron placas en transición en aorta. Las lesiones se caracterizaron por un incremento focal del espesor intimal causado por la infiltración de macrófagos espumosos y músculo liso, entremezclados en proporción variable con depósito de matriz extracelular (Figura 2). Los conejos que recibieron placebo no presentaron lesiones microscópicas en aorta.

Se ha demostrado que la cantidad de colesterol dietético y la duración de la dieta hipercolesterolémica modifican la morfología de la ateroesclerosis ${ }^{27}$. Conejos expuestos a altas dosis de colesterol (1 a $2 \%$ ) durante cortos períodos de tiempo ( 2 a 4 meses) desarrollan estrías grasas con abundantes macrófagos espumo$\operatorname{sos}^{2,4,19}$. Dietas con bajo contenido de colesterol $(0,15$ a $0,25 \%$ ) de larga duración ( 8 meses) ocasionan lesiones más complejas, compuestas por infiltración intimal de macrófagos, músculo liso y depósito de matriz extracelular hasta el desarrollo de ateromas: centro necrótico rico en lípidos y cristales de colesterol, recubierto por capa de tejido fibroso constituida por músculo liso, matriz extracelular y macrófagos espumosos ${ }^{16}$.

En nuestra experiencia, la administración de 0,62 a $1,24 \mathrm{~g} /$ día de colesterol durante 60 días indujo infiltración mixta de macrófagos espumosos y músculo liso en la íntima de la aorta, cambios que concuerdan con los estadios iniciales de la ateroesclerosis humana. En el ser humano, la ateroesclerosis comienza con la infiltración intimal de macrófagos cargados de vacuolas lipídicas ricas en colesterol (estría grasa) y progresa con la infiltración y proliferación de músculo liso, con síntesis de colágeno y matriz extracelular. Finalmente, se desarrolla una lesión compleja compuesta por un centro necrótico con restos celulares, cristales de colesterol y calcio, así como una envoltura fibrosa con células de músculo liso, macrófagos, células espumosas, linfocitos, colágeno y neovascularización (ateroma) 8, 9,23.

Histopatología de órganos. Todos los conejos que recibieron colesterol evidenciaron degeneración grasa hepática microvacuolar. Con la dosis de 0,62 g/día la degeneración grasa hepática fue de distribución centrolobulillar en 3 conejos y difusa, aunque más severa en región centrolobulillar, en los restantes animales. Con las dosis de 0,93 y $1,24 \mathrm{~g} /$ día todos los conejos presentaron degeneración grasa hepática difusa, más severa en la región centrolobulillar. La degeneración grasa hepática por dietas hipercolesterolémicas ha sido reportada en el conejo ${ }^{11,15,19}$, por lo cual se desaconseja la administración prolongada de este tipo de dietas para evitar mortalidad de animales por la hepatotoxicidad del colesterol ${ }^{16,27}$. Pese a esta restricción, el conejo alimentado con colesterol es uno de los modelos más empleados en ateroesclerosis experimental ${ }^{25,27}$.
También se constató infiltrado multifocal de macrófagos espumosos alrededor de capilares en coroides e iris de ojos (3 conejos del grupo 1; 6 conejos del grupo 2 y 6 conejos del grupo 3); plexos coroides de ventrículos laterales del cerebro (4 conejos del grupo 1; 4 conejos del grupo 2 y 6 animales del grupo 3); pulpa roja del bazo (6 conejos del grupo 2 y 6 conejos del grupo 3); intersticio renal (1 conejo del grupo 1; 6 conejos del grupo 2 y 6 animales del grupo 3) y lámina propia de mucosa bronquial (2 conejos del grupo 2). Los conejos del grupo control no presentaron lesiones en los órganos muestreados.

En conclusión, se confirma la extrema sensibilidad del conejo a desarrollar hipercolesterolemia y lesiones ateroescleróticas tempranas en aorta cuando es sometido a una dieta con altos niveles de colesterol. Las tres dosis de colesterol indujeron una severa hipercolesterolemia y lesiones ateroescleróticas de extensión variable en la aorta. Sin embargo, la dosis de 0,93 g/día durante 60 días fue la que produjo una respuesta hipercolesterolémica con menor variabilidad entre los conejos y lesiones ateroescleróticas bien desarrolladas con incremento del espesor intimal por infiltración de macrófagos espumosos, músculo liso y depósito variable de matriz extracelular en aorta. La estandarización de este modelo experimental de ateroesclerosis temprana permitirá estudiar el efecto ateroprotector de diferentes fármacos $\mathrm{y}$ alimentos funcionales.

\section{REFERENCIAS}

1. Adibi P, Sadeghi M, Mahsa M, Rozati G, Mhoseni M. 2007. Prediction of coronary atherosclerotic disease with liver transaminase level. Liver Int 27: 895-900.

2. Amran AA, Zakaria Z, Othman F, Das S, Raj S, Nordin M. 2010. Aqueosus extract of Piper sarmentosum decreases atherosclerotic lesions in high cholesterolemic experimental rabbits. Lipids Health Dis 9: 44-49.

3. Barnes SE, Weinberg PD. 1999. Two patterns of lipid deposition in the cholesterol fed rabbit. Arterioscler Thromb Vasc Biol 19: 2376-2386.

4. Cavallini DC, Abdalla DS, Vendramini RC, Vedan R, Bomdespacho LQ, Pauly ND, Valdez GF, Rossi EA. 2009. Effects of isoflavone-supplemented soy yogurt on lipid parameters and atherosclerosis development in hypercholesterolemic rabbits: a randomized double-blind study. Lipids Health Dis 8: 40-49.

5. Chi Z, Melendez AJ. 2007. Role of cell adhesion molecules and immune-cell migration in the initiation, onset and development of atherosclerosis. Cell Adhes Migrat 1: 171-175.

6. Cornhill JF, Barrett WA, Herderick EE, Mahley RW, Fry DL. 1985. Topographic study of sudanophilic lesions in cholesterol fed minipigs by image analysis. Arterioscler Thromb Vasc Biol 5: 415-426.

7. Dillard A, Matthan NR, Lichtenstein AH. 2010. Use of hamster as a model to study diet-induced atherosclerosis. Nutr Metab 7: 89-101. 
8. Galkina E, Ley K. 2009. Immune and inflammatory mechanisms of atherosclerosis. Ann Rev Immunol 27: 165197.

9. Glass FF, Witztum JL. 2001. Atherosclerosis: the road ahead. Cell 104: 503-516.

10. Goldbourt U, Neufeld HN. 1986. Genetic aspects of arteriosclerosis. Arterioscler Thromb Vasc Biol 6: 357-377.

11. Hakimoglu F, Kizil G, Kanay Z, Kizil M, Isi H. 2007. The effect of ethanol extract of Hypericum lysimachioides on lipid profile in hypercholesterolemic rabbits and its in vitro antioxidant activity. Atherosclerosis 192: 113-122.

12. Holman RL. 1958. Technic for studying atherosclerotic lesion. Lab Invest $7: 42-47$.

13. Jackson C, Benbow U, Bond A, Galley D, Schartz C. 2010. Animal models of atherosclerosis. In: Advances in Vascular Medicine (Abraham D, Handler C, Dashwood M, Coghlan G. Ed.), Springer, London, p.77-92.

14. Jawien J, Nastalek R, Korbut R. 2004. Mouse models of experimental atherosclerosis. J Physiol Pharmacol 55: 503-517.

15. Jayakody L, Kappagoda T, Senaratne MP, Thomson AB. 1988. Impairment of endothelium dependent relaxation: an early marker for atherosclerosis in the rabbit. $\mathrm{Br}$ J Pharmacol 94: 335-346.

16. Kolodgie FD, Katocs AS, Largis EE, Wrenn SM, Cornhill JF, Herderick EE, Lee SJ, Virmani R. 1996. Hypercholesterolemia in the rabbit induced by feeding graded amounts of low-level cholesterol. Arterioscler Thromb Vasc Biol 16: 1454-1464.

17. Kovanen PT, Brown MS, Basu SK, Bilheimer DW, Goldstein JL. 1981. Saturation and suppression of hepatic lipoprotein receptors: A mechanism for the hypercholesterolemia of cholesterol-fed rabbits. Proc Natl Acad Sci 78: 1396-1400.

18. Leite JO, DeOgburn R, Ratliff J, Su R, Smyth JA, Volek JS, McGrane MM, Dardik A, Fernandez ML. 2010. Low carbohydrate diets reduce lipid accumulation and arterial inflammation in guinea pigs fed a high-cholesterol diet. Atherosclerosis 209: 442-448.

19. Marinou KA. 2010. Differential effect of Psitacia vera extracts on experimental atherosclerosis in rabbit animal model: an experimental study. Lipids Health Dis 9: 73-81.
20. Moghadasian MH. 2002. Experimental atherosclerosis A historical overview. Life Sci 70: 855-865.

21. Organización Panamericana de la Salud. 2009. Indicadores Básicos. Argentina. http://publicaciones.ops.org.ar/ publicaciones/indicadores/indicadores_2009.pdf.

22. Schindhelm RK, Dekker JM, Nijpels G, Bouter LM, Stehouwer CD, Heine RJ, Diamant M. 2007. Alanine aminotransferase predicts coronary heart disease events: A 10-year follow-up of Hoorn Study. Atherosclerosis 191: 391-396.

23. Schoen FG. 2005. Vasos sanguíneos. En: Robbins y Cotran Patología Estructural y Funcional (Kummar V, Abbas A, Fausto N, ed), $7^{\mathrm{a}}$ ed, Elsevier, Madrid, p. 521-530.

24. Thiery J, Nebendhal K, Rapp K, Kluge R, Teupser D, Seidel D. 1995. Low atherosclerotic response of a strain of rabbits to diet-induced hypercholesterolemia. Arterioscler Thromb Vasc Biol 15: 1181-1188.

25. Verghese M, Richardson JE, Boateng J, Shackelford LA, Howard C, Walker LT, Chawan CB. 2008. Dietary lycopene has a protective effect on cardiovascular disease in New Zealand male rabbits. J Biol Sci 8: 268-277.

26. Woollard KJ. Greissmann F. 2010. Monocytes in atherosclerosis: subsets and functions. Nat Rev Cardiol 7: 77-96.

27. Yanni AE. 2004. The laboratory rabbit: an animal model of atherosclerosis research. Lab Anim 38: 246-256.

28. Yin W. 2012. Plasma lipid profiling across species for the identification of optimal animal model of human dyslipidemia. J Lipid Res 53: 51-65.

29. Yun KE, Shin CY, Yoon YS, Park HS. 2009. Elevated alanine aminotransferase levels predict mortality from cardiovascular disease and diabetes in Koreans. Atherosclerosis 205: 533-537.

30. Yuzefovych LV, Musiyenko SI, Wilson GL, Rachek LI. 2013. Mitochondrial DNA damage and dysfunction, and oxidative stress are associated with endoplasmic reticulum stress, protein degradation and apoptosis in high fat diet-induced insulin resistance mice. Plos One 8(1): e54059. 